\title{
Algorithm for management of an incarcerated inguinal hernia in the emergency settings with manual reduction. Taxis, the technique and its safety
}

\author{
M. Pawlak ${ }^{1}$ (D) B. East ${ }^{2,3} \cdot$ A. C. de Beaux ${ }^{4}$
}

Received: 28 November 2020 / Accepted: 11 May 2021 / Published online: 25 May 2021

(c) The Author(s), under exclusive licence to Springer-Verlag France SAS, part of Springer Nature 2021

\begin{abstract}
Background An acute inguinal hernia remains a common emergency surgical condition worldwide. While emergency surgery has a major role to play in treatment of acute hernias, not all patients are fit for emergency surgery, nor are facilities for such surgery always available. Taxis is the manual reduction of incarcerated tissues from the hernia sack to its natural compartment, and can help delay the need for surgery from days to months. The aim of this study was to prepare a safe algorithm for performing manual reduction of incarcerated inguinal hernias in adults.

Methods Medline, Scopus, Ovid and Embase were searched for papers related to emergency inguinal hernias and manual reduction. In addition, the British National Formulary and Safe Sedation Practice for Healthcare Procedures: Standards and Guidance were reviewed.

Results A safe technique of manual reduction of an acute inguinal hernia, called GPS (Gentle, Prepared and Safe) Taxis, is described. It should be performed within $24 \mathrm{~h}$ from the onset of a painful irreducible lump in groin, and when concomitant symptoms and signs of bowel strangulation are absent. Conscious sedation guidelines should be followed. The most popular drug combination is of intravenous morphine and short-acting benzodiazepine, both titrated carefully for optimal and safe effect. The dose of drugs must be individualised, and the smallest effective dosage should be used to avoid oversedation. Following successful taxis, the patient should undergo a short period of observation. Urgent surgery can be undertaken during the same admission or up to several weeks later.

Conclusions Taxis is a benign/non-invasive method for patients with an acute, non-strangulated inguinal hernias. It likely reduces the risk and complications of anaesthesia and surgery in the emergency settings. GPS Taxis should be considered as first line treatment in the majority of patients presenting with an acute inguinal hernia when existing bowel infarction is unlikely.
\end{abstract}

Keywords Taxis $\cdot$ Manual reduction $\cdot$ Inguinal hernia

\section{Introduction}

M. Pawlak

pawlakmd@gmail.com

1 Comprehensive Hernia Centre, North Devon District Hospital, Raleigh Heights, Barnstaple EX31 4JB, UK

2 3rd Department of Surgery at 1st Faculty of Medicine at Charles University, Motol University Hospital, Prague, Czech Republic

3 2nd Faculty of Medicine, Charles University, Prague, Czech Republic

4 Royal Infirmary of Edinburgh, Edinburgh, UK
Taxis is the manual, non-invasive, reduction of hernias combined with the administration of analgesia/sedation. The word 'Taxis' is derived from the Greek word meaning 'arrangement' as it describes the procedure of re-arranging the incarcerated hernia content back into the abdominal cavity [1]. Taxis was first discussed in the fourth century B.C. by the Greek Praxagoras of Cas [2]. Since then, a number of techniques have been described, but these appear to no longer be performed frequently, particularly in developed countries. Nevertheless, it is an important skill for all surgeons to be able to perform safely and appropriately. 
The acute inguinal hernia remains a common emergency surgical presentation, representing around $9 \%$ of inguinal hernia admissions [3]. While strangulation of the contents of the hernia is the main absolute indication for surgery, the majority of acutely symptomatic hernias actually do not have any strangulated contents, sometimes containing omentum only, or bowel which will remain viable if reduced promptly. If strangulation is excluded, and the hernia is able to be reduced, immediate surgery and indeed surgical admission can be avoided and hernia repair performed electively [4]. This is particularly useful in patients who would benefit from pre-optimisation, such as the correction of anticoagulation therapy. In addition, at times of altered surgical pathways due to pandemic outbreaks or war, reducing the need for emergency surgery has significant benefits [5].

30-day mortality after emergency inguinal surgery varies from 3.5 to $5 \%$ while it is around $0.07 \%$ for elective surgery $[6,7]$. However, these numbers do not take into consideration how many patients were treated with manual or spontaneous hernia reduction $[8,9]$ as only patients undergoing emergency surgery are being reported. Recently published International Guidelines on the management of groin hernia included a chapter on emergency management. This chapter focussed mainly on key questions around the best surgical approach (open anterior, posterior, or laparoscopic) and repair options (e.g. mesh versus non-mesh). Taxis, or the reduction of an acutely incarcerated hernia with the aid of analgesia/sedation and its safety was not covered [10, 11]. Similarly, the World Society of Emergency Surgery guidelines for emergency repair of complicated abdominal wall hernias and on Timing of Acute Care Surgery classification (TACS), only mention the situation of a strangulated hernia, which is recommended to need an immediate surgery, after recognition [11, 12].

The aim of this study was to describe the technique of taxis as we perform it, tips and tricks around its utilisation and most up to date information on sedation in patients with acute inguinal hernia presentation.

\section{Methods}

In March 2019 Medline, Scopus, Ovid and Embase were searched for papers related to emergency inguinal hernias. Search terms "groin", "inguinal", "strangulated", "incarcerated", "irreducible", "emergency", "groin/inguinal hernia", "taxis" and "reduction" were used. The nomenclature of the HerniaSurge Group was used to define 'Incarceration'- the inability to reduce the hernia mass into the abdomen; and 'Strangulation'- where the blood supply to the herniated tissues is compromised [10]. The relevant articles were examined against containing information on manual reduction. Those selected, together with authors' experience in the topic laid the groundwork for formulating the algorithm for performing manual reduction in incarcerated inguinal hernias. In addition, the British National Formulary was consulted for drug safety information regarding medications used for conscious sedation [13] as well as Safe Sedation Practice for Healthcare Procedures: Standards and Guidance [14].

\section{Results}

\section{Protocol}

The only real contra-indication to taxis is the presence of strangulated bowel within the hernia $[4,15]$. Thus recognising the likelihood of this is very important. Features suggestive of strangulation of the hernia contents include tachycardia, hypotension, or peritonitis. Localised signs in the region of the hernia, such as erythema, with the skin hot and painful to touch are also suggestive of strangulation. Differential diagnosis of a painful swelling in the groin must be considered. Other causes including abscess, testicular torsion, hydrocele and enlarged inflamed lymph nodes should be excluded, especially if the patient does not report the presence of a hernia prior to the onset of the current events $[16,17]$. Keep in mind that testicular torsion is a surgical emergency just as urgent if not more as strangulated hernia.

Blood markers for bowel ischaemia include serum D-dimer $>300 \mathrm{ng} / \mathrm{ml}$ (sensitivity $85 \%$, specificity $41 \%$ and negative predictive value $94 \%$ ) and serum phosphokinase levels of $140 \mathrm{IU} / 1$ and higher (compared to 90 in the control group) [18]. However, these are not used in routine practice. The white cell count, serum lactate, amylase and C-reactive protein levels had little predictive value with regard to bowel ischaemia in relation to an inguinal hernia $[15,18]$.

There appears to be a linear relationship between the time from the onset of symptoms, to strangulation and the mortality rate [15]. Two time frames were distinguished. First, up to $24 \mathrm{~h}$ from the onset of symptoms (not the time of admission), that is pain, firmness and inability to reduce the content of the inguinal hernia with simple manipulation, but minimal if any localised signs overlying the hernia. In this early stage, after conscientious examination and assessing the risk of strangulation as unlikely, taxis can be attempted. After $24 \mathrm{~h}$ from the onset of symptoms, (and each further $24 \mathrm{~h}$ ) the risk of hernia content strangulation and ischaemia doubles [15], surgical treatment with bowel viability verification should be the first line of treatment. However, like so much in surgery, careful assessment of each case is necessary, and time from onset of symptoms is a rough guide rather than a specific cut off point in decision making.

Symptoms and signs of bowel obstruction alone without significant localised or systemic signs do not seem to be 
a contra-indication to taxis. Abdominal pain or pain that persists in the region of the hernia after taxis is a concern that taxis has not been successful, or reduction-en-masse has occurred, a condition when the hernia contents and sac are 'reduced' but the hernia sac contents remains incarcerated within the hernia sac $[19,20]$.

An algorithm for the management of the acute incarcerated inguinal hernia in adults is given in Fig. 1.

\section{Technique}

To perform the manual reduction of an incarcerated inguinal hernia successfully the focus is on three key elements. For the purpose of easy recollection, the acronym of "GPS Taxis" was coined.

\section{GPS}

Be Gentle: it is not a forceful direct pressure on the sack as often the hernia contents will mushroom over the external ring and not reduce. It is a gentle manipulation to introduce the contents back into abdominal cavity through the hernial ring.

Be Prepared: appropriate early resuscitation. Administer oxygen therapy. Establish intravenous access. Use vital sign monitoring, with a minimum of oxygen saturation and heart rate. The environment should be quiet, away from a noisy emergency department for example. The bed, should be able to be put into the Trendelenburg position. If the patient is old and frail, the presence of an anaesthetist is suggested.

Be Safe: if strangulation of the bowel is likely then taxis is not appropriate! Use the smallest effective dosage of analgesia and sedation when attempting taxis.

\section{Analgesia and sedation}

Taxis involves conscious sedation using a combination of drugs to reduce pain (analgesia) and to aid relaxation (sedation). The most popular drug regimen described in the literature for manual reduction of inguinal hernias is intravenous diazepam and morphine [14, 21-23]. However, in more recent times midazolam with morphine is becoming a more popular combination [14].

Taxis does take time to perform safely. Understanding the pharmacokinetics of any drugs given makes the technique safer. Avoid the intramuscular route, as drug absorption can be variable. The intravenous route is preferred, with intravenous access maintained at all times. Intravenous morphine can take 5-10 min to take effect [24]. It is best titrated, giving $1 \mathrm{mg}$ boluses every minute until pain symptoms improve [14]. Intravenous midazolam has a faster onset of action but it still may take 5 or so minutes to reach good effect [25]. Again, $1 \mathrm{mg}$ per minute boluses once the morphine is given is recommended [14]. Dilution of the drugs with normal saline makes the inadvertent bolus of more than $1 \mathrm{mg}$ per minute less likely. Remember, the onset of action is delayed with both drugs in low cardiac output states.

A key statement is that the dosage of these drugs must be individualized, and the smallest effective dosage should be used $[14,25]$. This is especially true in geriatric or debilitated patients, low cardiac states, liver disease or low serum albumin. Typical doses of morphine are 5-10 mg (0.1-0.15 mg/kg), and midazolam/diazepam, 2.5-5 mg [13].

Regular communication with the patient, in addition to putting them at ease, allows monitoring of the level of sedation. Monitoring should be continued into the recovery period [14], because of the duration of action of the analgesia/sedation medication.
Fig. 1 Management of the acute incarcerated inguinal hernia in adults. GPS Taxis: $G$ be gentle. A constant, steady pressure over the hernia for $5-15 \mathrm{~min}$, avoiding forceful pushing. $P$ be prepared. Appropriate environment with oxygen therapy, oxygen saturation and pulse monitoring, opioid and benzodiazepine antagonists at the bed side. $S$ be safe. Careful titration of intra-venous opioids + benzodiazepines

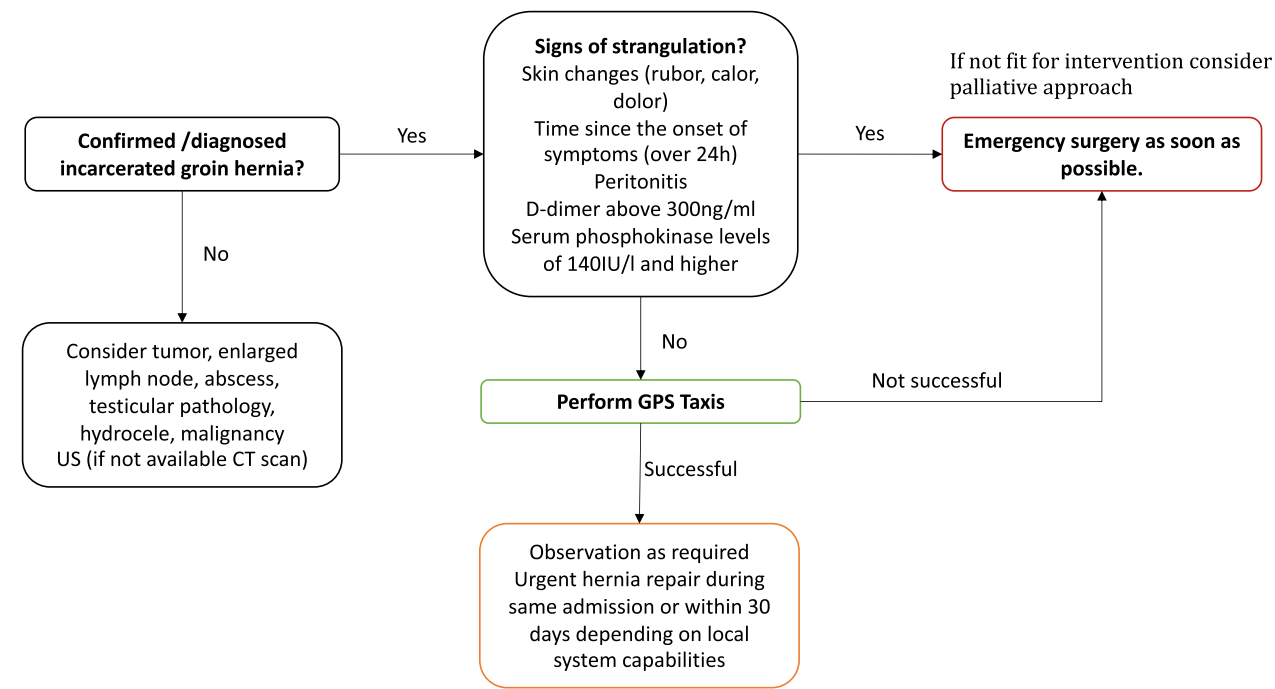


The primary adverse effect of morphine is hypotension. Respiratory suppression can also occur, and its risk increases with the co-administration of sedative agents. Naloxone is used to reverse opiate overdose-initially 100-200 $\mu \mathrm{g}$ $(1.5-3 \mu \mathrm{g} / \mathrm{kg})$ by bolus intravenous injection. Since naloxone has a shorter duration of action than many opiates, close monitoring and repeated administration of naloxone as necessary according to the respiratory rate and depth of coma is often required [13].

Midazolam is a benzodiazepine of choice for use in conscious sedation as it is fast- and short-acting, which make it safer than for example, Diazepam [21]. The effect of such longer acting drugs is unpredictable and the drugs may show unwanted interactions, which are difficult to control [22]. Nevertheless, diazepam does have some benefits over midazolam for its additional central muscle-relaxant effects which may be of benefit during taxis [2]. Benzodiazepines are contra-indicated in acute pulmonary insufficiency and marked neuromuscular respiratory weakness. Overdose of benzodiazepines is usually manifested by central nervous system depression ranging from drowsiness to coma. Initially, central nervous system stimulation with perioral tingling may be present. Flumazenil, is a reversal agent for benzodiazepines, given as an intravenous bolus at $0.01 \mathrm{mg} /$ $\mathrm{kg}$ up to $2 \mathrm{mg}$ over $15 \mathrm{~s}$ [13].

Remember the term is 'conscious sedation'. Give the drugs slowly titrating the response. Remember the time for onset of action. Monitoring of the patient post-taxis is important, as this is often the time when consciousness may be lost, and resuscitation may be required. Always check and adhere to your local conscious sedation guidelines or ask anaesthetist to help you and best to be present during the procedure.

\section{Manual reduction}

Manual hernia reduction is performed using both hands with the patient in as much Trendelenburg position as tolerated. First, the fascial defect should be identified by gentle palpation around the base of the incarcerated hernia sack.

It is important to keep in mind the 3D anatomy of the inguinal canal. Direct hernias are easier to reduce but indirect hernias can easily lead to the pressure being applied in wrong direction. Some authors describe gentle pulling on the distal part of the sac first in a direction away from the body $[26,27]$. This allows the contents to align themselves in the direction of the canal which will make gentle reposition of the hernia's contents back into the abdominal cavity easier. This manoeuvre can be painful as indeed can the whole manipulation and every abdominal contraction due to pain makes the reduction more difficult or even impossible. That is why it is important to work slowly and gently and if possible, administer the sedation/analgesia titrating its effect to achieve a degree of comfort and thus relaxation of the abdominal wall.

The non-dominant hand can be used for "pulling" the hernia and making it longer and narrower while the dominant hand is gently pressing on or more encircling the contents of the incarcerated hernia, almost massaging it bit by bit into the abdominal cavity. If there is any bowel present, an air bubble may be palpated and pressure on this area will make the contents smaller and more easy to reduce.

This gentle pressure can take 5-10 $\mathrm{min}$, and should not be a forceful push (so as to prevent en-mass reduction or bowel perforation). If bowel is in the hernial sac, reduction is often achieved with a satisfying gurgling sound [4, 15, 16, 28, 29].

The chance of reducing bowel that is already irreversibly ischaemic is very unlikely. In the proposed algorithm, it is suggested that following successful taxis a necessary period of observation takes place ensuring recovery of the patient from the effects of any sedation/analgesia. The patient should meet standard discharge criteria. Urgent elective hernia repair can then be planned within 30 days or considered at the initial admission [4]. Worsening pain, deteriorating clinical observations and/or signs mandate further investigation or surgical intervention with visualization of the bowel. Laparoscopy through the hernia sack is a useful adjunct in open surgery, or conventional laparoscopy [30, 31].

An ultrasound guided taxis may increase the success rate of taxis alone with the need for emergency surgery decreasing from $10 \%$ to $2.0 \%$ and makes the assessment of the contents of the hernia and its viability possible [16].

\section{Discussion}

Taxis is a useful skill to be able to perform safely when access to emergency surgery is compromised. The recent COVID-19 pandemic has highlighted this need. A safe environment, safe drug administration and careful approach to taxis are the key steps for safe reduction of an acute hernia.

The level of medical evidence relating to the emergency management of groin hernias is low. There appears to have been a shift from taxis to emergency inguinal hernia surgery over the last century. Yet a more recent cohort study published in 2009, reported manual reduction of incarcerated hernia with a success rate was $70 \%$ with no taxis-related complications during the follow-up period [4]. Reduction of infarcted bowel appears to be very unlikely with GPS Taxis [4, 16, 31-33], as does reduction-en-masse. But taxis is not something to attempt with all acute inguinal hernias. If there is strong suspicion of bowel infarction, resuscitation and emergency surgical repair is the mainstay of management $[11,12]$.

It is unclear if the patient can be discharged within hours of successful taxis or if a period of observation is necessary. 
Any effects of the analgesia/sedation should have worn off. And the ease of the taxis may help decide the safe observation period-the longer and more forceful the need to achieve taxis, the longer the patient should be observed. The time of day and frailty of the patient may be other factors that determine post-taxis discharge planning.

The surgical approach to the patient with an acute hernia that has failed taxis is outside the scope of this study. However, in a patient who appears to have had successful taxis, but then deteriorates over 12-24 h, and the working diagnosis is bowel ischaemia, laparoscopy, if possible, is the logical next step. This can be performed through the hernia sac, or by more conventional laparoscopy $[31,33]$.

Ultrasound guided taxis in one study reduced the need for emergency hernia repair fivefold [16]. However, this technique does not appear to be in common practice. Nevertheless, ultrasound is a useful first line investigation when the diagnosis is unclear.

\section{Limitation}

The study describes a technique that was once well recognised but has largely been abandoned due to changes in strategy towards emergency hernia surgery. Therefore, recent, relevant data are scarce. However, large registry data could help inform on this technique.

\section{Conclusions}

1. Manual reduction is a benign/non-invasive method for patients with short history of incarcerated inguinal hernia, up to $24 \mathrm{~h}$ from the onset of symptoms.

2. Attention to detail of the technique of taxis likely improves success rate and minimises morbidity, e.g. reduction of non-viable bowel and en-masse reduction.

3. GPS Taxis should be a tool in the tool box of every general surgeon involved in emergency care.

Supplementary Information The online version contains supplementary material available at https://doi.org/10.1007/s10029-021-02429-1.

Author contributions All authors contributed to the study conception and design. Material preparation, data collection and analysis were performed by MP, BE and AdB. The first draft of the manuscript was written by MP and all authors contributed equally to final versions of the manuscript. All authors read and approved the final manuscript.

Funding The research has not been funded.

Code availability No software application or costume code is available for this study.

\section{Declarations}

Conflict of interest MP, BE and $\mathrm{ACdB}$ have no conflicts of interest.

Ethical approval This study was performed in line with the principles of the Declaration of Helsinki. The study was exempt from ethics committee approval.

Informed Consent Not applicable.

Human and animal rights Not applicable.

\section{References:}

1. O'Toole MT (2013) Mosby's medical dictionary, 9th edn. Elsevier

2. Garg G, Jenaw R, Chaudhary S, Garg KM (2014) How safe is taxis in hernia? Reduction of gangrenous omentum-an uncommon occurrence. IOSR J Dent Med Sci 13:52-53. https://doi.org/10. 1007/s10029-008-0467-9

3. Primatesta P, Goldacre MJ (1996) Inguinal hernia repair: incidence of elective and emergency surgery, readmission and mortality. Int J Epidemiol 25:835-839

4. Harissis HV, Douitsis E, Fatouros M (2009) Incarcerated hernia: to reduce or not to reduce? Hernia 13:263-266. https://doi.org/10. 1007/s10029-008-0467-9

5. Stabilini C, East B, Fortelny R et al (2020) European Hernia Society (EHS) guidance for the management of adult patients with a hernia during the COVID-19 pandemic general concepts. Hernia 1:3. https://doi.org/10.1007/s10029-020-02212-8

6. Nilsson E, Kald A, Anderberg B et al (1997) Hernia surgery in a defined population: a prospective three year audit. Eur J Surg 163:823-829

7. Cheek CM, Primatesta P, Goldacre N (1997) Inguinal hernia repair: incidence of elective and emergency surgery, readmission and mortality. Int J Epidemiol 26:459-461

8. Montgomery J, Dimick JB, Telem DA (2018) Management of groin hernias in adults-2018. JAMA 320:1029. https://doi.org/ 10.1001/jama.2018.10680

9. Hernández-Irizarry R, Zendejas B, Ramirez T et al (2012) Trends in emergent inguinal hernia surgery in Olmsted County, MN: a population-based study. Hernia 16:397-403. https://doi.org/10. 1007/s10029-012-0926-1

10. Simons MP, Smietanski M, Bonjer HJ et al (2018) International guidelines for groin hernia management. Hernia 22:1-165. https:// doi.org/10.1007/s10029-017-1668-x

11. Birindelli A, Sartelli M, Di Saverio S et al (2017) 2017 update of the WSES guidelines for emergency repair of complicated abdominal wall hernias. World J Emerg Surg 12:37. https://doi. org/10.1186/s13017-017-0149-y

12. Kluger Y, Ben-Ishay O, Sartelli M et al (2013) World society of emergency surgery study group initiative on timing of acute care surgery classification (TACS). World J Emerg Surg 8:1-6. https:// doi.org/10.1186/1749-7922-8-17

13. BNF: British National Formulary-NICE. https://bnf.nice.org.uk/ drug/. Accessed 25 Dec 2019

14. Academy of Medical Royal Colleges (2013) Safe Sedation Practice for Healthcare Procedures: Standards and Guidance I The Royal College of Anaesthetists. https://www.aomrc.org.uk/wpcontent/uploads/2016/05/Safe_Sedation_Practice_1213.pdf

15. Koizumi M, Sata N, Kaneda Y et al (2014) Optimal timeline for emergency surgery in patients with strangulated groin hernias. Hernia 18:845-848. https://doi.org/10.1007/s10029-014-1219-7 
16. Chen S-C, Lee C-C, Liu Y-P et al (2005) Ultrasound may decrease the emergency surgery rate of incarcerated inguinal hernia. Scand J Gastroenterol 40:721-724. https://doi.org/10.1080/0036552051 0015485

17. LeBlanc KA, Kingsnorth A, Sanders DL (2018) Management of abdominal hernias. Springer International Publishing

18. Icoz G, Makay O, Sozbilen M et al (2006) Is D-dimer a predictor of strangulated intestinal hernia? World J Surg 30:2165-2169. https://doi.org/10.1007/s00268-006-0138-x

19. Wu SC, Wang CC, Sheen-Chen SM (2008) Intestinal obstruction caused by intraoperative mass reduction of an incarcerated inguinal hernia-report of a case. Int J Surg 6:e103-e105. https:// doi.org/10.1016/j.ijsu.2007.04.017

20. Arima T, Muroya K, Kawamoto K et al (2018) Laparoscopic relief of reduction en masse followed by elective preperitoneal inguinal hernia repair with modified Kugel ${ }^{\mathrm{TM}}$ patch. Int J Surg Case Rep 50:97-99. https://doi.org/10.1016/j.ijscr.2018.07.026

21. Wright SW, Chudnofsky CR, Dronen SC et al (1993) Comparison of midazolam and diazepam for conscious sedation in the emergency department. Ann Emerg Med 22:201-205. https://doi.org/ 10.1016/S0196-0644(05)80203-3

22. Section and Board of Anaesthesiology EU of MS (2007) Guidelines for sedation and/or analgesia by non-anaesthesiology doctors. Eur J Anaesthesiol 24:646-647. https://doi.org/10.1017/ S0265021507000452

23. Benger J, Gray A, Lloyd G, Norris A, Strachan R, Telford LGR (2012) Safe sedation of adults in the emergency department. Royal College of Anaesthetists

24. Aubrun F, Mazoit J-X, Riou B (2012) Postoperative intravenous morphine titration. Br J Anaesth. https://doi.org/10.1093/bja/ aer458

25. Malamed SF (2005) Sedation: a guide to patient management. Anesth Prog 52:43-44

26. Brunicardi F et al (1980) Schwartz's principles of surgery, 10th edn. McGraw Hill Education
27. Henry K (1938) Hernia: anatomy, etiology, symptoms, diagnosis, differential diagnosis, prognosis, and the operative and injection treatment. By Leigh F. Watson, M.D., Member of Attending Staff of California Lutheran Hospital and Methodist Hospital of Southern California. Br J Surg 26:452-452. https://doi.org/10.1002/bjs. 18002610234

28. Pérez JAÁ, Baldonedo RF, Bear IG et al (2003) Emergency hernia repairs in elderly patients. Int Surg 88:231-237

29. Yang XF, Liu JL (2014) Acute incarcerated external abdominal hernia. Ann Transl Med 2:1-9. https://doi.org/10.3978/j.issn. 2305-5839.2014.11.05

30. Koivusalo A, Pakarinen MP, Rintala RJ (2007) Laparoscopic herniorrhaphy after manual reduction of incarcerated inguinal hernia. Surg Endosc 21:2147-2149. https://doi.org/10.1007/ s00464-007-9318-5

31. Sgourakis G, Radtke A, Sotiropoulos GC et al (2009) Assessment of strangulated content of the spontaneously reduced inguinal hernia via hernia sac laparoscopy: preliminary results of a prospective randomized study. Surg Laparosc Endosc Percutan Tech 19:133-137. https://doi.org/10.1097/SLE.0b013e31819d8b8b

32. Sawayama H, Kanemitsu K, Okuma T et al (2014) Safety of polypropylene mesh for incarcerated groin and obturator hernias: a retrospective study of 110 patients. Hernia 18:399-406. https:// doi.org/10.1007/s10029-013-1058-y

33. Tebala GD, Kola-Adejumo A, Yee J (2019) Hernioscopy: a reliable method to explore the abdominal cavity in incarcerated or strangulated inguinal hernias spontaneously reduced after general anaesthesia. Hernia 23:403-406. https://doi.org/10.1007/ s10029-019-01901-3

Publisher's Note Springer Nature remains neutral with regard to jurisdictional claims in published maps and institutional affiliations. 